\title{
Tilt-to-Length Coupling in the GRACE Follow-On Laser Ranging Interferometer
}

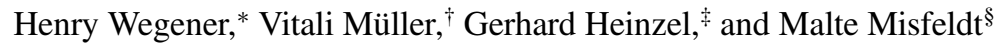 \\ Max Planck Institute for Gravitational Physics (Albert Einstein Institute), 30167 Hannover, Germany
}

https://doi.org/10.2514/1.A34790

\begin{abstract}
The Laser Ranging Interferometer onboard the Gravity Recovery and Climate Experiment Follow-On satellites is the first laser interferometer in space measuring satellite-to-satellite distance variations. One of its main noise sources at low frequencies is the so-called tilt-to-length coupling, caused by satellite pointing variations. This error is estimated by fitting a linear coupling model, making use of the so-called center-of-mass calibration maneuvers. These maneuvers are performed regularly for the original purpose of center-of-mass determination. Here, the results of the tilt-to-length estimations for the Laser Ranging Interferometer are presented in terms of coupling factors, which are all within $200 \mu \mathrm{m} \cdot \mathrm{rad}^{-1}$ and thus meet the requirements. From these parameters, estimations of nadir and cross-track components of the spacecraft center-of-mass positions with respect to the interferometer reference point are derived, providing an additional method to track center-of-mass movement over time.
\end{abstract}

\section{Nomenclature}

$\boldsymbol{p}=$ spacecraft positions, $\mathrm{m}$
$\boldsymbol{q}=$ spacecraft attitude quaternions
$\boldsymbol{X}=$ spacecraft state vector
$\theta=$ intersatellite pointing angles, $\mathrm{rad}$
$\lambda=$ tilt-to-length coupling factors, $\mathrm{m} \cdot \mathrm{rad}^{-1}$
$\rho=$ intersatellite range, $\mathrm{m}$
$\boldsymbol{\omega}=$ spacecraft angular velocities, $\mathrm{rad} \cdot \mathrm{s}^{-1}$

\section{Introduction}

$\mathbf{T}$ HE Gravity Recovery and Climate Experiment (GRACE) [1-4] successfully measured the time-variable gravity field of Eart $\bar{h}$, from 2002 until 2017, far beyond its planned lifetime of 5 years. The successor mission GRACE Follow-On (GRACE-FO) [5], launched in May 2018, was designed as an almost identical copy of GRACE: two satellites, about $200 \mathrm{~km}$ apart, one trailing the other in a nearpolar orbit at approximately $500 \mathrm{~km}$ altitude. The main science instrument in both missions is a $\mathrm{K} / \mathrm{Ka}$-band microwave ranging (KBR) system, measuring the biased range (i.e., distance variations) between the two satellites [6]. Additionally, GRACE-FO is equipped with the Laser Ranging Interferometer (LRI), which measures the same observable as the KBR, but with higher accuracy, and serves as a technology demonstrator [7-10].

The LRI is a heterodyne laser interferometer operating with a wavelength of $1064.5 \mathrm{~nm}$ [7]. At each spacecraft $(\mathrm{S} / \mathrm{C})$, the laser light is reflected by a triple mirror assembly (TMA), consisting of three mirrors in a corner cube reflector arrangement [11-13]. The three mirror planes are pairwise perpendicular, and their virtual intersection point is called the vertex point (VP). It was shown in [14] that the LRI measures distance changes between the two VPs, one at each S/C. Consequently, the measured range is invariant under small rotations of either one of the S/C around its VP, which is, to the accuracy of S/C integration, placed in the center of mass (c.m.) of the

Received 24 February 2020; revision received 26 May 2020; accepted for publication 10 June 2020; published online Open Access 27 July 2020. Copyright $\odot 2020$ by the American Institute of Aeronautics and Astronautics, Inc. All rights reserved. All requests for copying and permission to reprint should be submitted to CCC at www.copyright.com; employ the eISSN 1533-6794 to initiate your request. See also AIAA Rights and Permissions www.aiaa.org/randp

*Research Scientist, Callinstr. 36/38; also Leibniz Universität Hannover, 30167 Hannover, Germany.

†Senior Research Scientist, Callinstr. 36/38; also Leibniz Universität Hannover, 30167 Hannover, Germany.

†Professor, Callinstr. 36/38; also Leibniz Universität Hannover, 30167 Hannover, Germany.

${ }^{\S}$ Research Scientist, Callinstr. 36/38; also Leibniz Universität Hannover, 30167 Hannover, Germany.
S/C. Because of residual offsets between the VP and c.m. of the order of $100 \mu \mathrm{m}$, there is a difference between the distance from VP to VP on the one hand, and the distance from c.m. to c.m. on the other hand. This difference is varying, when the $\mathrm{S} / \mathrm{C}$ rotates around its c.m. (cf. Fig. 1). Hence, $\mathrm{S} / \mathrm{C}$ attitude variations cause an error in the measured biased range. This effect is called tilt-to-length (TTL) coupling. There are also other smaller mechanisms that cause TTL, such as the laser beam passing through beam splitters on the optical bench.

The conservative preflight total noise budget of the LRI of $80 \mathrm{~nm} \sqrt{\mathrm{Hz}}$ at high frequencies (cf. [8] ) comprises the rms sum of laser frequency noise $(40 \mathrm{~nm} \sqrt{\mathrm{Hz}})$, TTL $(40 \mathrm{~nm} \sqrt{\mathrm{Hz}})$, thermal effects and geometric path-length changes $(30 \mathrm{~nm} \sqrt{\mathrm{Hz}})$, and several sources of readout noise $(3 \mathrm{~nm} \sqrt{\mathrm{Hz}})$, plus a top-level margin. At frequencies below $35 \mathrm{mHz}$, the LRI range spectrum is dominated by the gravity signal, and at high frequencies by laser frequency noise, with less than $1 \mathrm{~nm} \sqrt{\mathrm{Hz}}$ above $200 \mathrm{mHz}$ significantly below the requirements [7]. A detailed analysis of the real data, done by the authors, has by now revealed that, between 35 and $200 \mathrm{mHz}$, ranging variations due to nongravitational forces govern the LRI range spectrum.

Tilt to length is a common error source for laser interferometers in space, such as the LISA Pathfinder mission [15] and the LISA mission [16], which makes it an important topic of study. For gravity field recovery from GRACE-FO observations, error sources, such as accelerometer (ACC) noise or temporal aliasing errors, are dominating, while the ranging accuracy has less influence [17]. Our results are thus not only meant to provide a means of mitigating the TTL error for the LRI, but also with respect to future applications of other instruments of its kind. The findings presented here characterize for the first time the TTL error for intersatellite laser interferometers in orbit, and we show that the LRI fulfills its requirements.

Among other instruments, each satellite carries an ACC manufactured by ONERA-The French Aerospace Lab $[\underline{18}, 19]$ to remove the nongravitational acceleration contributions from the range in the process of gravity field recovery [1]. To ensure the correct measurement of accelerations, the c.m. of the proof mass of this ACC must be colocated with the S/C c.m., with a requirement for the displacement of $0.5 \mathrm{~mm}$ in each direction and a requirement for stability of $0.1 \mathrm{~mm}$ per half-year [20]. Because of residual offsets, S/C rotations couple into the measured linear accelerations. For the purpose of correcting this error, so-called c.m. calibration (CMC) maneuvers are scheduled regularly. The satellites are commanded to perform small periodic rotations, individually, around one axis at a time. By fitting a model to the linear accelerations that are induced by these rotations, the c.m. positions of the GRACE-FO satellites with respect to the ACC reference point can be estimated [20,21]. Occasionally, the S/C c.m. are shifted back to the ACC reference point by moving trim masses on the satellites. This process is called mass trim maneuver. 


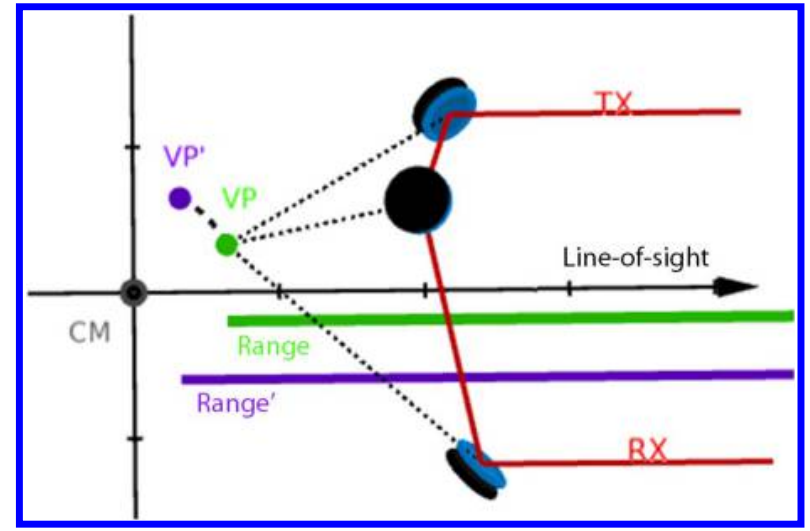

Fig. 1 Rotation of the VP around the c.m. varies the measured range, approximated by the projection onto the line of sight.

In this paper, we show how the LRI TTL coupling can be calibrated by making use of these CMC maneuvers. First, the CMC maneuvers are briefly described with emphasis on what has changed from GRACE to GRACE-FO. We outline the most important steps of data processing and the different sources of $S / C$ attitude information. Then, different methods of parameter estimation are presented, as well as the model for the TTL ranging error as a function of the intersatellite pointing angles (roll, pitch, and yaw), which depends on six model parameters, the linear coupling factors. In the last section, we present and discuss the results of the coupling factor estimation. Furthermore, we derive nadir and cross-track components of the S/C c.m. positions with respect to the VPs.

\section{Satellite Rotation Maneuvers}

The CMC maneuvers on GRACE-FO are performed using magnetic torque rods, whose main purpose is to control the $\mathrm{S} / \mathrm{C}$ attitude in the nominal mission phase [22]. These rods, three per $\mathrm{S} / \mathrm{C}$, produce a magnetic dipole moment, which causes a torque in combination with the Earth's magnetic field. It is noteworthy that they do not induce a linear acceleration on the $\mathrm{S} / \mathrm{C}$ in contrast to control thruster firings. Each maneuver is meant to cause angular motion around one of the S/C axes, in this context called roll, pitch, and yaw axes. For the GRACE mission, roll and pitch maneuvers were executed near the North Pole within the region between $60^{\circ}$ and $80^{\circ}$ latitude; yaw maneuvers were executed near the equator between $10^{\circ}$ and $20^{\circ}$ latitude [21]. These locations were carefully chosen. One of the main criteria for planning the maneuvers is that the achievable magnitude of the angular acceleration is maximal in one of the $\mathrm{S} / \mathrm{C}$ axes, whereas low in the other axes.

The optimal maneuver locations for GRACE-FO are similar to those for GRACE because both missions use a near-polar orbit. However, the achievable magnitude of the angular accelerations has decreased due to two reasons. First, the mass of the satellites has been increased from roughly $475 \mathrm{~kg}$ per S/C for GRACE [23] to $601 \mathrm{~kg}$ mass at launch for GRACE-FO [24]. Second, the maximum input current for the magnetic torque rods has been lowered from 120 to $110 \mathrm{~mA}$, such that each rod on GRACE-FO is able to produce a magnetic dipole moment of $27.5 \mathrm{~A} \cdot \mathrm{m}^{2}$, as opposed to $30.0 \mathrm{~A} \cdot \mathrm{m}^{2}$ on GRACE [25]. According to our simulations based on these specifics, the largest achievable angular accelerations for GRACEFO are roughly $16.3,3.3$, and $2.9 \mu \mathrm{rad} \cdot \mathrm{s}^{-2}$ for roll, pitch, and yaw axes, respectively. The respective values for GRACE were 25.6, 5.5, and $4.8 \mu \mathrm{rad} \cdot \mathrm{s}^{-2}$.

Ideally, only one of the pointing angles is excited, while rotations around the other two axes are kept small to avoid correlations between the resulting pointing angles. Considering this and other criteria discussed in [21], the magnitudes of the angular accelerations achieved in the recent $\mathrm{CMC}$ maneuvers are up to about 12.4, 2.3, and $1.4 \mu \mathrm{rad} \cdot \mathrm{s}^{-2}$ for roll, pitch, and yaw axes. This results in pointing angle oscillations with amplitudes about $50,10,6 \mu \mathrm{rad} \cdot \mathrm{s}^{-2}$, respectively, at a maneuver frequency of $83 . \overline{3} \mathrm{mHz}$, on top of the natural pointing deviations. Figure $\underline{2}$ shows pointing angles during a roll maneuver (top left), a pitch maneuver (top right), and a yaw maneuver (bottom) performed on GRACE-FO 1 (GF1) on 23 June 2019. The pointing angles for this plot were computed from IMU1B RL04 data [24] by integrating angular velocities, as described next, in the section on pointing angles. All angles were filtered with a passband from 50 to $120 \mathrm{mHz}$ to isolate the signal at the maneuver frequency from the total pointing deviations, which can be as large as several milliradians after $180 \mathrm{~s}$ without the nominal attitude control mode. Various sources for pointing angles, their exact definition, and the data processing are described in the following section.

The excitation of the roll angle during the yaw maneuver is not desired, but unavoidable. The yaw excitation is achieved by activating the torque rod in the $y$ direction of the satellite frame (SF), which is defined in [24]. This inevitably produces a torque in SF $x$ direction due to Earth's magnetic field component in SF $z$ direction. This $z$ component is rapidly changing near the equator, which explains the decreasing roll angle amplitude in Fig. 2.

During a CMC maneuver on GRACE-FO, two of the torque rods are activated in such a way that the resulting magnetic dipole moment is aligned perpendicular to the desired rotation axis. Its magnitude profile is rectangular with a period of $12 \mathrm{~s}$, yielding the highest signal at a Fourier frequency of $83.3 \mathrm{mHz}$. Each CMC maneuver consists of 15 cycles of this profile, which results in a maneuver duration of $180 \mathrm{~s}$. The nearly square wave pattern allows for larger angle amplitudes compared to a sine wave pattern. Center-of-mass calibration maneuvers have been performed once per month up to September 2019 , and hereafter will be performed in a half-yearly cycle. At each instance, 14 maneuvers in total (i.e., seven maneuvers per S/C) are spread over two calendar days. For each of the angles roll, pitch, and yaw, two maneuvers are performed. Furthermore, one mixed maneuver per $\mathrm{S} / \mathrm{C}$ is performed, exciting both roll and pitch angles. The same procedure was used for GRACE, however, with a slightly different magnitude profile.

In principle, the c.m. determination can be carried out using CMC maneuvers simultaneously performed on both $\mathrm{S} / \mathrm{C}$, because the measurements of one ACC are independent of the rotations of the distant S/C. Regarding the LRI, the TTL error caused by the pointing variations of the two $\mathrm{S} / \mathrm{C}$ couple into the same measurement, the biased range. If both $\mathrm{S} / \mathrm{C}$ simultaneously perform rotation maneuvers, it may not be possible to disentangle the two effects, which would likely result in less accurate parameter estimation. For this reason, CMC maneuvers on GRACE-FO are planned, such that the time spans of any two maneuvers do not overlap. Because the accuracy of TTL estimation turns out to be sufficient, as described in this paper, no dedicated TTL calibration maneuvers are planned for the GRACE-FO LRI.

\section{Data Processing}

\section{A. LRI Range}

The LRI telemetry data contain the raw optical beat-note phase measured in cycles. The downlinked data are sampled at roughly $10 \mathrm{~Hz}$. The phase contains occasional unintended jumps, which are correlated to attitude control thruster activations. The jumps are removed, and thus do not influence the performance of the LRI [7]. The time tags of the ranging data have to be corrected for clock errors and additionally for a deterministic delay due to onboard decimation filtering. The phase measured on the transponder S/C is subtracted from the master phase, after jumps have been removed from both measurements and after the two data streams have been synchronized. Subsequently, the combined phase is converted from cycles to biased range in meters. Before the estimation of TTL coupling factors, the range is filtered using a band-pass filter with cutoff frequencies at 50 and $120 \mathrm{mHz}$. The same filter is applied to the time series of the pointing angles.

Attitude control thruster firings cause a residual linear acceleration, in addition to the intended angular acceleration. The range variations in line-of-sight direction of such a linear acceleration are measured by the LRI and can disturb the analysis of CMC maneuvers. During a maneuver, attitude control thrusters are deactivated on the 

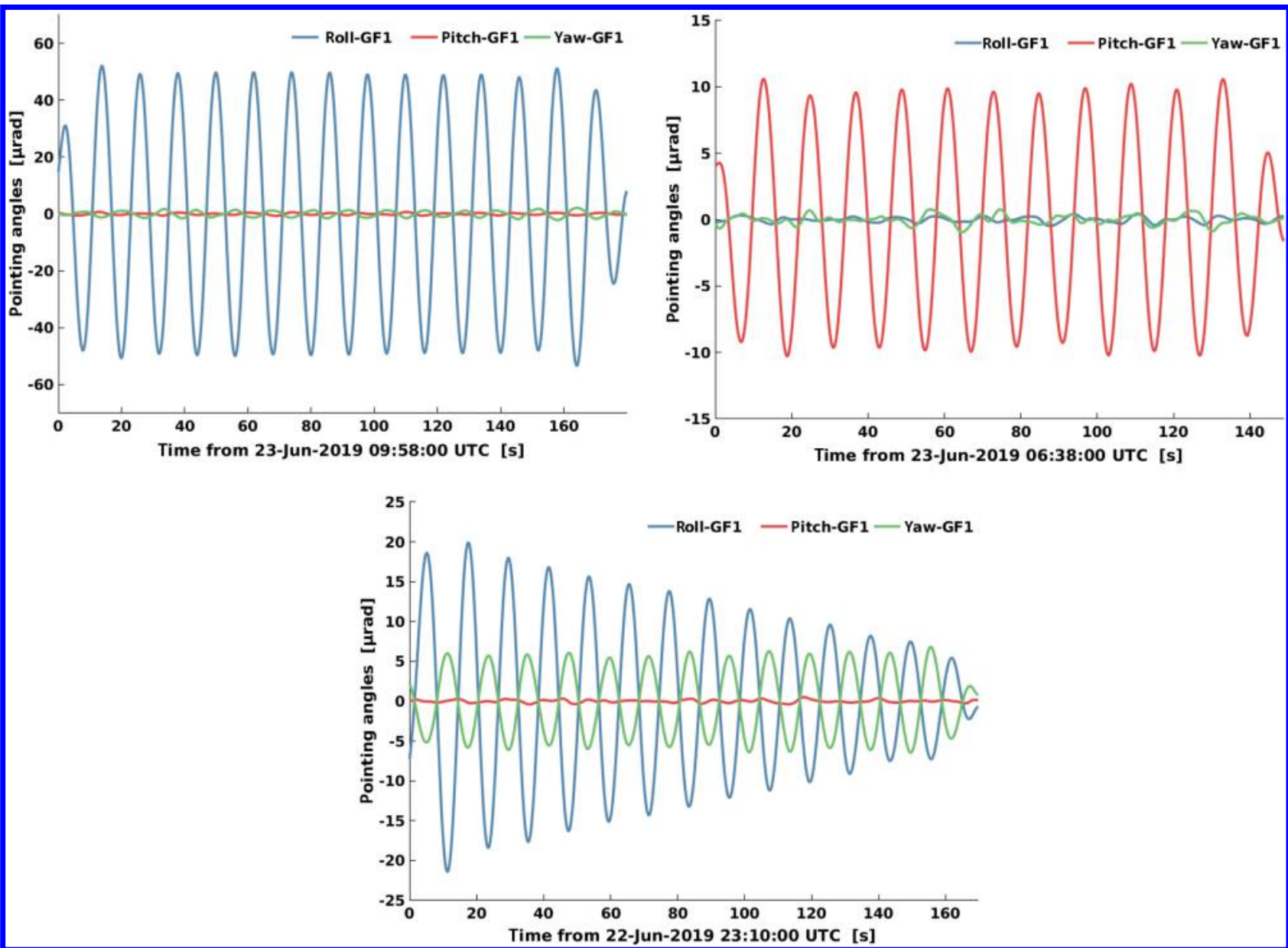

Fig. 2 Band-pass-filtered satellite pointing angles during CMC maneuvers on GF1 in June 2019; roll maneuver (top left); pitch maneuver (top right); yaw maneuver (bottom).

S/C performing the maneuver. However, they can occur on the other $\mathrm{S} / \mathrm{C}$. There are two options to cope with this effect. First, the respective data segment can be excluded from the TTL analysis. With this approach, about $84 \%$ of the CMC maneuver data could be used. A second option is to remove the range variations caused by thruster firings from the LRI data. To this end, we have derived a model of this effect from data segments without maneuvers, using thruster activation times and durations provided in the THR1B data product [24]. With the range adjusted in this way, about $90 \%$ of the maneuver data

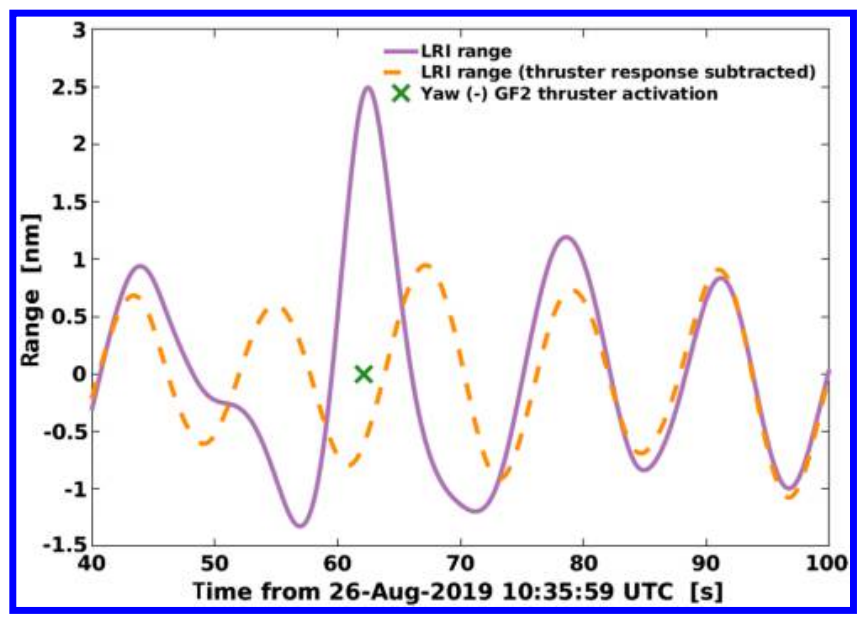

Fig. 3 Band-pass-filtered LRI range with and without subtraction of the thruster response. could be used for TTL estimation. Figure $\underline{3}$ illustrates an example of this subtraction for a yaw GF2 thruster event during a pitch GF1 maneuver performed on 26 August 2019. The green cross marks the beginning of the thruster activation, which, in this case, has a duration of $50 \mathrm{~ms}$. Our model for each specific thruster consists of a constant linear acceleration (i.e., with a rectangular shape), with the exact duration provided in the THR1B data, and a single model parameter, which describes the magnitude of the acceleration. The respective LRI response is obtained by applying decimation filter of the LRI and a lowpass filter. Additionally, the time series shown in Fig. 3 is filtered once more, using the band-pass filter described previously. Any filtering is performed twice, in two ways, to compensate for the filter delay. This explains why the effect of the thruster activation is visible before the actual activation. Our derived model of these linear accelerations is consistent with the models that were used to compute the ACT1B data product [26], that is, our obtained values for the acceleration magnitude are close to those reported in [26] for the along-track direction.

\section{B. Satellite Pointing Angles}

The intersatellite pointing deviations can be described by the three Euler angles of the rotation, which transforms between the SF and the line-of-sight frame (LOSF) [27]. In this context, the angles of rotation around SF $x, y$, and $z$ axes are called roll, pitch, and yaw angles, respectively. Here, the SF $x$ axis is roughly aligned with the line of sight, the SF $y$ axis is pointing to the right of the S/C, and the SF $z$ axis is roughly the nadir direction. The definition of the $\mathrm{SF}$ is given in [24].

Let $p$ denote the position of the $\mathrm{S} / \mathrm{C}$ in inertial Earth-centered frame. Denoting by $\boldsymbol{p}_{\text {other }}$ the position of the other S/C, the LOSF is defined as 


$$
\begin{aligned}
& \boldsymbol{X}_{\mathrm{LOSF}}=\frac{\boldsymbol{p}_{\mathrm{other}}-\boldsymbol{p}}{\left|\boldsymbol{p}_{\mathrm{other}}-\boldsymbol{p}\right|} \\
& \boldsymbol{Y}_{\mathrm{LOSF}}=\boldsymbol{X}_{\mathrm{LOSF}} \times \frac{\boldsymbol{p}}{|\boldsymbol{p}|} \\
& \boldsymbol{Z}_{\mathrm{LOSF}}=\boldsymbol{X}_{\mathrm{LOSF}} \times \boldsymbol{Y}_{\mathrm{LOSF}}
\end{aligned}
$$

The pointing angles, which we use, are defined by

$$
R_{\mathrm{SF}}^{\mathrm{LOSF}}=R_{z}\left(\theta_{z}\right) R_{y}\left(\theta_{y}\right) R_{x}\left(\theta_{x}\right)
$$

where $R_{x}\left(\theta_{x}\right)$ denotes a rotation around the $x$ axis by an angle $\theta_{x}$, which we will call the roll angle. $R_{y}\left(\theta_{y}\right)$ and $R_{z}\left(\theta_{z}\right)$ denote rotations around the $y$ and $z$ axes, by angles $\theta_{y}$ and $\theta_{z}$, called pitch and yaw, respectively. $R_{\mathrm{SF}}^{\mathrm{LOSF}}$ denotes the rotation, which transforms a vector given in SF to its representation in LOSF.

GRACE-FO hosts a variety of science instruments, whose measurements can be used to derive the pointing angles: LRI fast steering mirror (FSM), star camera assembly (SCA), ACC, and inertial measurement unit (IMU). The FSM provides pitch and yaw angles independently of other sensors. Computing pointing angles from the other measurements requires to compute the LOSF axes, which can be done using GPS-derived S/C positions. Then, the SF axes are obtained from the $\mathrm{S} / \mathrm{C}$ attitude quaternions. The quaternions can be taken directly from SCA data, or integrated using data from other attitude sensors and the dynamics equations for the 7-D state vector $\boldsymbol{X}$ :

$$
\boldsymbol{X}=\left(\begin{array}{c}
\boldsymbol{X}_{q} \\
\boldsymbol{X}_{\omega}
\end{array}\right)=\left(\begin{array}{c}
\boldsymbol{q} \\
\boldsymbol{\omega}
\end{array}\right)
$$

with derivatives ([28] p. $519 \mathrm{ff})$

$$
\dot{\boldsymbol{X}}_{q}=\frac{1}{2} \Omega(\omega) \cdot \boldsymbol{q}
$$

and

$$
\dot{X}_{\omega}=J^{-1}(\tau-\omega \times(J \omega))
$$

The notation for these equations is explained in the Appendix. Once LOSF and SF axes are known, pointing angles can be derived from Eq. (1). In the following, we briefly describe the different attitude sensors.

\section{Fast Steering Mirror}

In the nominal fine-pointing mode, the $\mathrm{S} / \mathrm{C}$ attitude and orbit control system keeps the pointing angles within a few hundred microradians from their nominal values. The LRI requires even more accurate pointing of the laser beam, which is ensured by the use of a steering mirror on the optical bench, controlled by a feedback loop using the differential wave-front sensing technique [7]. The steering mirror orientation is downlinked at the same sampling rates as the LRI phase measurements and can be directly converted to Euler angles referring to the LRI optical frame, which is defined in [24], and then to pitch and yaw angles referring to the SF. The steering mirror does not provide roll angles. The resolution of the measured and downlinked pitch and yaw angles is limited by quantization noise of the steering mirror readout to about $1 \mu \mathrm{rad} \sqrt{\mathrm{Hz}}$ for each axis.

\section{Star Camera Assembly}

The quaternions from the SCA1B data describe the rotation between the SF and the inertial frame. In the current release RL04 of Level-1B data, SCA1B data contain quaternions generated from SCA1A and IMU1A, which are fused using a Kalman filter [24]. Without using IMU data, angles derived from star camera quaternions have significantly higher noise (cf. Fig. 4). Even the fused attitude solution for the pitch angle seems to have a slightly elevated spectrum for Fourier frequencies between 10 and $100 \mathrm{mHz}$.

We report that there seems to be a mismatch of approximately $1 \mathrm{~s}$ between the time stamps of the pointing angles derived from SCA1B
RL04 data on the one hand, and other attitude sensor data on the other hand. Therefore, we have subtracted $1 \mathrm{~s}$ from the time stamps of the SCA1B RL04 data, before the TTL estimation.

\section{Inertial Measurement Unit}

Each GRACE-FO satellite carries an Astrix 120 IMU manufactured by Airbus Defence and Space [23]. Each IMU hosts four laser fiber gyroscopes, measuring the angular velocities in four different axes. These angular velocities can be converted to the SF and integrated, using Eq. (2). Initial values can be taken from SCA data. The obtained quaternions can be used to derive pointing angles from Eq. (1). Because of error propagation in the integration process, this is only useful for a relatively short time or when fused with other sensors. We use the IMU1B RL04 data product.

\section{Accelerometer}

The ACC onboard of each S/C also measures angular accelerations. These can be integrated using Eqs. (2) and (3) to obtain quaternions (cf. [28]). From these quaternions, the pointing angles can be derived in the same manner as from the star camera quaternions. Because of error propagation in the integration process, this is only useful for a very short time or when fused with other sensors. However, the noise at higher Fourier frequencies, in particular at the CMC maneuver frequency of $83 . \overline{3} \mathrm{mHz}$, is very low. We use the ACC1A RL04 data product merely to analyze its suitability for LRI TTL estimation. The ACC on the second satellite (GF2) has been switched to a coarser operation mode (large range mode) and is currently not providing data with sufficient accuracy [26].

Figures $\underline{4}$ and $\underline{5}$ examplarily show a spectrum and a time series of the pitch and yaw angles of GF1, derived from different sensors. The data span a period of $1000 \mathrm{~s}$ on 14 January 2019. The spectra were computed using the logarithmic frequency axis power spectral density method [29]. The amplitude spectral densities derived from this method are abbreviated LASD. SCA1B RL04 data are attitude quaternions fused from SCA1A and IMU1A data [24]. "SCA only" data refer to attitude quaternions derived merely from SCA1A data, without using IMU data, provided to us by Jet Propulsion Laboratory.

Figure $\underline{6}$ compares the LRI range to the total TTL error, computed using coupling factors estimated from the CMC maneuvers in June 2019, as described as follows (see Table 1). The pointing angles were derived from different sources. The SCĀ $1 \mathrm{~B}$ time stamps have been adjusted by subtracting $1 \mathrm{~s}$, as explained previously. In principle, any of these four sources is capable of providing pointing angles for TTL estimation. However, we do not use the ACC data to produce the final estimation of the TTL coupling factors because the GF2 ACC has been switched to a coarser operation mode. In the following section, we will describe the model and the parameter estimation methods that we used to estimate the TTL coupling factors.

\section{Parameter Estimation}

\section{A. TTL Model}

Let us write the measured range as $\hat{\rho}=\rho+\delta_{\mathrm{TTL}}+\delta_{\text {other }}$, where $\rho$ is the true distance between the satellite c.m.; $\delta_{\mathrm{TTL}}$ is the TTL error caused by the VP c.m. offsets of both S/C; and $\delta_{\text {other }}$ denotes the contribution from all other error sources, including a bias. Using the subscript $i \in\{1,2\}$ to indicate satellite 1 (GF1) or satellite 2 (GF2), let $\boldsymbol{V} \boldsymbol{P}_{i}$ and $\boldsymbol{C} \boldsymbol{M}_{i}$ denote the positions of TMA VPs and S/C c.m., respectively. Furthermore, let $\boldsymbol{e}_{\mathrm{VP}, i}$ be the unit vector pointing from the VP on S/C $i$ toward the VP on the other S/C, and let $\boldsymbol{e}_{\mathrm{LOS}, i}$ be the unit vector pointing from the c.m. on $\mathrm{S} / \mathrm{C} i$ toward the c.m. on the other S/C. With this notation, we have

$$
\begin{aligned}
& \hat{\rho}=\left|\boldsymbol{V} \boldsymbol{P}_{2}-\boldsymbol{V} \boldsymbol{P}_{1}\right|+\delta_{\text {other }}=\boldsymbol{e}_{\mathrm{VP}, 1} \cdot\left(\boldsymbol{V} \boldsymbol{P}_{2}-\boldsymbol{V} \boldsymbol{P}_{2}\right)+\delta_{\text {other }} \\
& \rho=\left|\boldsymbol{C} \boldsymbol{M}_{2}-\boldsymbol{C} \boldsymbol{M}_{1}\right|=e_{\mathrm{LOS}, 1} \cdot\left(\boldsymbol{C} \boldsymbol{M}_{2}-\boldsymbol{C} \boldsymbol{M}_{1}\right)
\end{aligned}
$$

For S/C $i$, let $\boldsymbol{V}_{i}$ be the vector pointing from the TMAVP to the S/C c.m. For $\rho \gg\left|V_{i}\right|, i=1,2$, the approximation $\boldsymbol{e}_{\mathrm{VP}, i} \approx \boldsymbol{e}_{\mathrm{LOS}, i}$ yields 

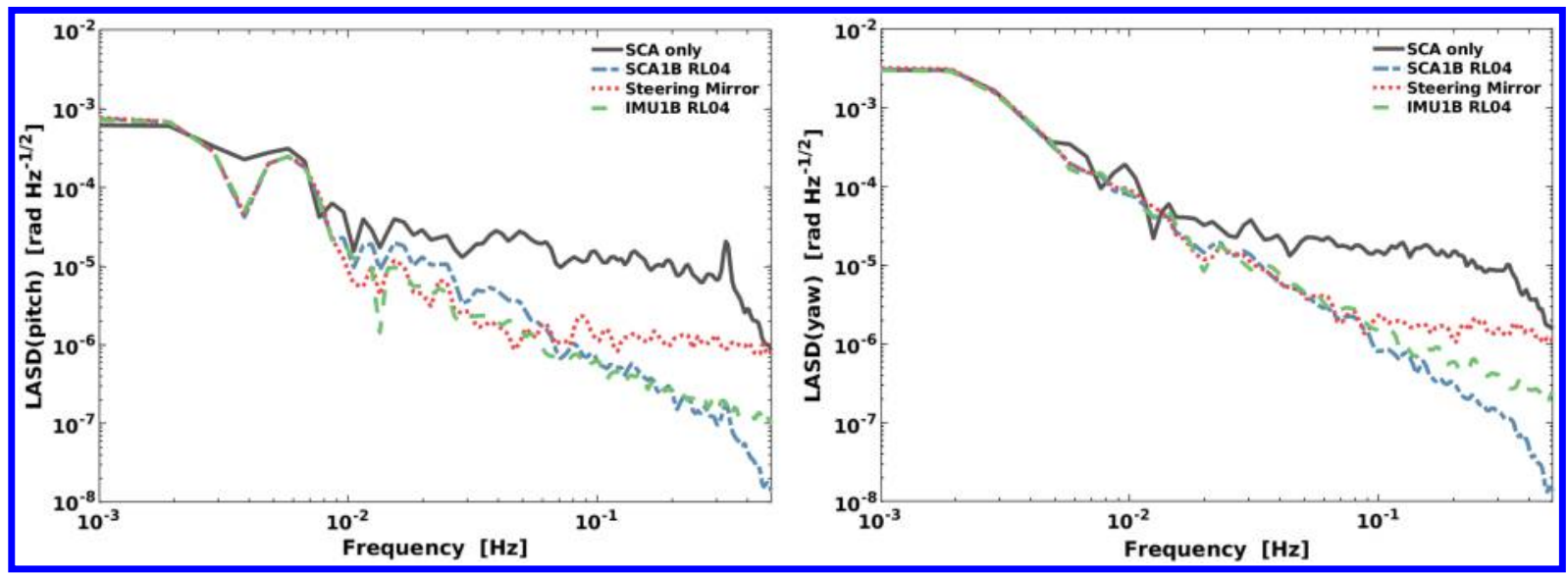

Fig. 4 Amplitude spectral density of pointing angles, computed from the $1000 \mathrm{~s}$ time series depicted in Fig. $\underline{.}$.
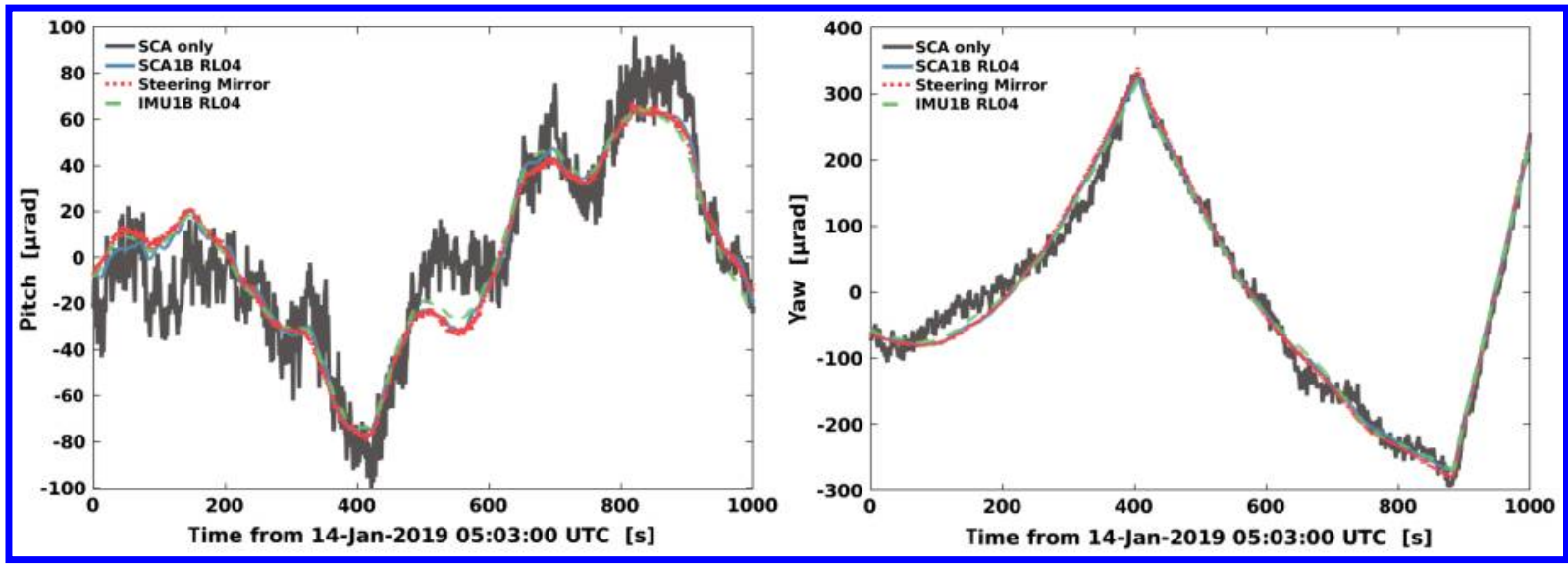

Fig. 5 Pointing angles of GF1, based on different data types; here, for a period of $1000 \mathrm{~s}$ on 14 January 2019; pitch (left); yaw (right).

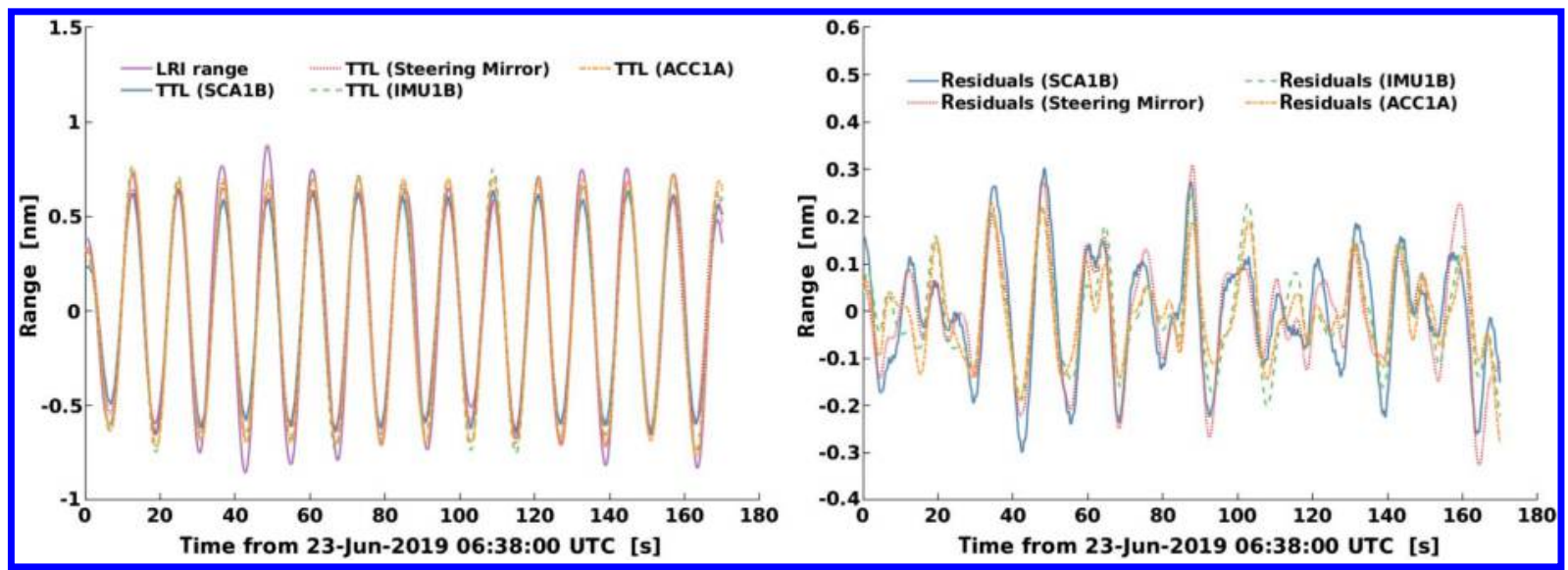

Fig. 6 Range vs. estimated TTL error (left) and residuals (right) from different attitude sources, during a pitch GF1 maneuver on 23 June 2019.

$$
\begin{aligned}
\delta_{\mathrm{TTL}}=\hat{\rho}-\rho-\delta_{\mathrm{other}} & \approx \boldsymbol{e}_{\mathrm{LOS}, 1} \cdot\left(\boldsymbol{V} \boldsymbol{P}_{2}-\boldsymbol{V} \boldsymbol{P}_{1}-\boldsymbol{C} \boldsymbol{M}_{2}+\boldsymbol{C} \boldsymbol{M}_{1}\right) \\
& =\boldsymbol{e}_{\mathrm{LOS}, 1} \cdot\left(\boldsymbol{V}_{1}-\boldsymbol{V}_{2}\right) \\
& =\boldsymbol{e}_{\mathrm{LOS}, 1} \cdot \boldsymbol{V}_{1}+\boldsymbol{e}_{\mathrm{LOS}, 2} \cdot \boldsymbol{V}_{2}
\end{aligned}
$$

$$
\begin{aligned}
\delta_{\mathrm{TTL}, i} & =\boldsymbol{e}_{\mathrm{LOS}}^{\mathrm{LOSF}} \cdot \boldsymbol{V}^{\mathrm{LOSF}} \\
& =\left(\begin{array}{lll}
1 & 0 & 0
\end{array}\right) \cdot R_{\mathrm{SF}}^{\mathrm{LOSF}} \cdot \boldsymbol{V}^{\mathrm{SF}} \\
& =R_{11} \Delta x+R_{12} \Delta y+R_{13} \Delta z
\end{aligned}
$$

where $R_{\alpha \beta}$ is the entry in row $\alpha$ and column $\beta$ of $R_{\mathrm{SF}}^{\mathrm{LOSF}}$. Vector superscripts indicate the frame in which it is expressed, for example, $\boldsymbol{V}^{\mathrm{SF}}=(\Delta x, \Delta y, \Delta z)^{T}$ is the notation of $\boldsymbol{V}$ with components in the SF. In terms of pointing angles, writing $c_{x}=\cos \left(\theta_{x}\right), s_{x}=\sin \left(\theta_{x}\right)$, etc., using Eq. (1), we have 
Table 1 Estimated linear TTL coupling factors

\begin{tabular}{|c|c|c|c|c|c|c|}
\hline \multirow[b]{5}{*}{ Date } & \multicolumn{6}{|c|}{ Requirements on coupling factors, $\mu \mathrm{m} \cdot \mathrm{rad}^{-1}$} \\
\hline & 20 & 200 & 200 & 20 & 200 & 200 \\
\hline & \multicolumn{6}{|c|}{ Estimated LRI linear TTL coupling factors, $\mu \mathrm{m} \cdot \mathrm{rad}^{-1}$} \\
\hline & \multicolumn{3}{|c|}{ GF1 } & \multicolumn{3}{|c|}{ GF2 } \\
\hline & $c_{\text {roll }}$ & $c_{\text {pitch }}=\Delta z_{1} \cdot \mathrm{rad}^{-1}$ & $c_{\text {yaw }}=-\Delta y_{1} \cdot \operatorname{rad}^{-1}$ & $d_{\text {roll }}$ & $d_{\text {pitch }}=\Delta z_{2} \cdot \operatorname{rad}^{-1}$ & $d_{\text {yaw }}=-\Delta y_{2} \cdot \operatorname{rad}^{-1}$ \\
\hline $\begin{array}{l}\text { Wiggle maneuvers } \\
21 \text { June } 2018\end{array}$ & $0.4 \pm 7.1$ & $116.5 \pm 14.5$ & $154.1 \pm 23.0$ & $-5.9 \pm 7.7$ & $68.7 \pm 12.0$ & $82.8 \pm 37.5$ \\
\hline CMC maneuvers & & & & & & \\
\hline $\begin{array}{l}16 \text { Jan. } 2019 \\
26 \text { March } 2019\end{array}$ & $\begin{array}{l}0.2 \pm 1.0 \\
1.0 \pm 1.1\end{array}$ & $\begin{array}{l}90.8 \pm 9.6 \\
83.3 \pm 8.2\end{array}$ & $\begin{array}{l}62.6 \pm 44.5 \\
70.6 \pm 58.1\end{array}$ & $\begin{array}{l}-0.1 \pm 1.7 \\
-0.4 \pm 1.2\end{array}$ & $\begin{array}{l}74.9 \pm 9.9 \\
74.4 \pm 8.9\end{array}$ & $\begin{array}{l}142.7 \pm 17.6 \\
138.3 \pm 22.2\end{array}$ \\
\hline 24 April 2019 & $0.8 \pm 1.6$ & $90.9 \pm 10.0$ & $82.8 \pm 33.6$ & $-1.1 \pm 1.5$ & $83.9 \pm 9.8$ & $132.5 \pm 16.5$ \\
\hline 23 May 2019 & $1.0 \pm 0.9$ & $82.9 \pm 9.2$ & $90.3 \pm 32.2$ & $-1.0 \pm 1.1$ & $46.6 \pm 7.5$ & $103.2 \pm 22.5$ \\
\hline 22 June 2019 & $-0.4 \pm 1.2$ & $91.5 \pm 8.6$ & $98.8 \pm 31.3$ & $0.4 \pm 1.0$ & $61.0 \pm 54.7$ & $97.1 \pm 13.9$ \\
\hline 24 July 2019 & $2.2 \pm 1.5$ & $99.2 \pm 11.3$ & $94.9 \pm 33.0$ & $2.5 \pm 1.2$ & $73.7 \pm 9.0$ & $133.4 \pm 17.0$ \\
\hline 26 Aug. 2019 & $1.3 \pm 0.8$ & $94.9 \pm 14.0$ & $94.5 \pm 36.4$ & $1.1 \pm 0.9$ & $98.7 \pm 11.2$ & $133.9 \pm 19.6$ \\
\hline 28 Sept. 2019 & $0.4 \pm 1.4$ & $100.7 \pm 10.8$ & $86.0 \pm 30.3$ & $1.3 \pm 0.9$ & $93.5 \pm 11.1$ & $155.9 \pm 32.0$ \\
\hline
\end{tabular}

Average over different methods and different sources for the pointing angles. For each S/C, the coupling factors for pitch and yaw can be interpreted as offsets in SF $y$ and SF $z$ directions between S/C c.m. and LRI reference point, using Eq. (4).

$$
\delta_{\mathrm{TTL}, i} \approx c_{y} c_{z} \Delta x+\left(s_{x} s_{y} c_{z}-c_{x} s_{z}\right) \Delta y+\left(c_{x} s_{y} c_{z}+s_{x} s_{z}\right) \Delta z
$$

The second-order approximation for small angles is

$\delta_{\mathrm{TTL}, i} \approx\left(1-\frac{1}{2} \theta_{y}^{2}-\frac{1}{2} \theta_{z}^{2}\right) \cdot \Delta x+\left(\theta_{x} \theta_{y}-\theta_{z}\right) \cdot \Delta y+\left(\theta_{y}+\theta_{x} \theta_{z}\right) \cdot \Delta z$

The constant term can be neglected because the LRI is measuring the biased range, that is, the measured phase is ambiguous by an integer number of cycles. Adding up the terms for both $\mathrm{S} / \mathrm{C}$, the firstorder approximation of the total TTL error due to VP c.m. offsets is

$$
\delta_{\mathrm{TTL}}=\delta_{\mathrm{TTL}, 1}+\delta_{\mathrm{TTL}, 2} \approx \Delta z_{1} \theta_{y, 1}-\Delta y_{1} \theta_{z, 1}+\Delta z_{2} \theta_{y, 2}-\Delta y_{2} \theta_{z, 2}
$$

where $\theta_{y, i}$ and $\theta_{z, i}$ stand for pitch and yaw angles of S/C $i$, respectively. $\Delta y_{i}$ and $\Delta z_{i}$ denote $y$ and $z$ components of the vector pointing from the TMA VP to the c.m. of S/C $i$, respectively, expressed in the SF. In particular, we do not expect linear coupling of the roll angles. To verify this hypothesis and to monitor possible change, we estimate linear roll coupling factors, nevertheless. This yields in total six model parameters: the TTL coupling factors $\mathrm{CF}=\left(c_{\text {roll }}, c_{\text {pitch }}, c_{\text {yaw }}, d_{\text {roll }}, d_{\text {pitch }}, d_{\text {yaw }}\right)$. From Eq. (4), we expect $\mathrm{CF} \approx\left(0, \Delta z_{1},-\Delta y_{1}, 0, \Delta z_{2},-\Delta y_{2}\right)$.

Potential sources of TTL coupling other than the geometrical VP c.m. offset have been identified and studied extensively before the launch of GRACE-FO $[\underline{8}, \underline{14}, \underline{30}]$. For example, the optical path length through some components on the LRI optical bench can depend on the incident beam angle. The resulting TTL coupling is expected to be quadratic in the pitch and yaw angles and of the order of 2 and $4 \mathrm{~mm} \cdot \mathrm{rad}^{-2}$, respectively. In the nominal operation mode, the pitch and yaw angles are controlled to stay in a range of \pm 200 and $400 \mu \mathrm{rad}$, respectively. Linearizing the quadratic coupling around these values yields maximal linear TTL coupling of 0.8 and $3.2 \mu \mathrm{m}$. $\operatorname{rad}^{-1}$ for pitch and yaw, respectively.

Because of imperfect knowledge of the alignment of different reference frames, it may only be possible to determine the pointing angles up to a constant. In this sense, the pointing angles vary around some constant values, which we call here a static pointing offset. For linear coupling, such a static pointing offset leads to a constant pathlength error, which is irrelevant for the biased range. For quadratic coupling, however, it leads to a nonconstant path-length error and thus cannot be ignored. Figure 7 shows a spectrum of the LRI range and the linear TTL error, computed from a 9 day data segment (9-17 April 2019). The shown TTL error was derived using pointing

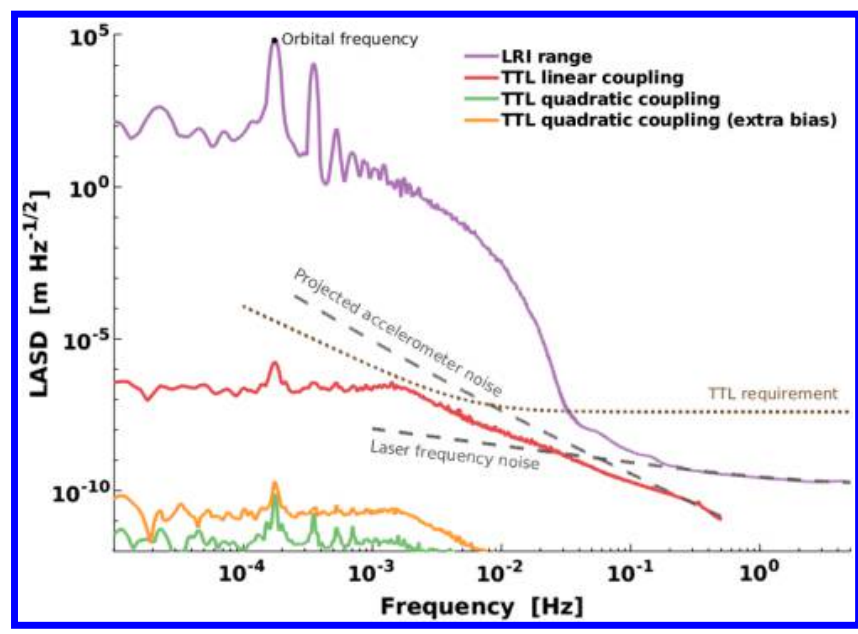

Fig. 7 Amplitude spectral density of LRI range, measured linear TTL coupling, and expected quadratic TTL coupling.

information from SCA1B RL04 data and with coupling factors obtained from the CMC maneuvers performed on 24-25 April 2019. In relation to the other error contributions, the assumed quadratic coupling of $2 \mathrm{~mm} \cdot \mathrm{rad}^{-2}$ for pitch and $4 \mathrm{~mm} \cdot \mathrm{rad}^{-2}$ for yaw is negligible (green line), even if an additional bias of $1 \mathrm{mrad}$ is assumed for all pointing angles (orange line).

For comparison, Fig. 7 also shows a model of LRI laser frequency noise derived from ground measurements and ACC noise based on a model for GRACE [31], converted to line-of-sight range. Noise in the ACC data is one of the error contributors in gravity field recovery. Figure 7 illustrates that the contribution from TTL is lower than the contribütion from ACC noise. The elevated LRI range spectrum between 40 and $200 \mathrm{mHz}$ is caused by physical range variations from nongravitational forces, which can be partly removed using ACC data [32]. The preflight noise budget allocated for TTL coupling is depicted by the dotted brown line, that is, $40 \mathrm{~nm} \sqrt{\mathrm{Hz}}$ times a noiseshape function, which is given in [7].

\section{B. Methods of Parameter Estimation}

We used five different methods to estimate the TTL coupling factors, all based on the band-pass-filtered time series of the range and the pointing angles, recorded during the execution of the CMC maneuvers. We distinguish two kinds of methods, as described as follows: 
1) With these methods, the TTL coupling factors of all six pointing angles are estimated at once, using the data from all $14 \mathrm{CMC}$ maneuvers.

a) LSQ: The pointing angles are fitted to the range using linear least squares. Denoting by $\theta$ the matrix of pointing angles $(N \times 6)$ and by $y$ the time series of the range $(N \times 1)$, where $N$ is the number of samples, the estimator is defined as

$$
\hat{\lambda}=\left(\theta^{T} \theta\right)^{-1} \theta^{T} y
$$

and its standard deviation is

$$
\sigma(\hat{\lambda})=\sigma\left(\delta_{\text {other }}\right) \cdot \operatorname{diag}\left(\sqrt{\left|\left(\theta^{T} \theta\right)^{-1}\right|}\right)
$$

where $\sigma\left(\delta_{\text {other }}\right)$ is the standard deviation of the ranging noise without TTL. After band-pass filtering around the maneuver frequency of $83.3 \mathrm{mHz}, \sigma\left(\delta_{\text {other }}\right)$ is of the order of $1 \mathrm{~nm}$.

b) BAY: The probability density of the coupling factors is computed using a Bayesian approach, which is sketched subsequent to this listing of methods.

2) These methods use merely the pitch and yaw maneuvers, which make up 8 out of the 14 CMC maneuvers. For each individual maneuver, the coupling factor for the associated angle is estimated. Because there are two pitch and two yaw maneuvers per S/C, we obtain two values for each coupling factor, which is finally estimated by taking the mean of the two values. Roll and mixed maneuvers are ignored by these methods, and roll coupling factors are not estimated.

a) LSI: The respective pointing angle is fitted to the range using linear least squares. The estimator is defined as for the LSQ method, but with only one pointing angle instead of six. b) PSD: The amplitude spectra of the range and the respective pointing angle are computed from the time series. Both spectra show peaks at the maneuver frequency of $83 . \overline{3} \mathrm{mHz}$. The magnitude of the coupling factor is estimated by the quotient of the height of the peaks. Its sign is determined by minimizing the residual. The spectra were computed using the MATLAB toolbox LTPDA, and we chose a flattop window (HFT90D) with a good amplitude accuracy [33].

c) XC: The cross correlation of the range with the pointing angle is computed. The cross-correlation $\chi_{\max }$ with maximal absolute value is determined. Here, we restrict the search to a maximal time shift of $2.5 \mathrm{~s}$, and we use the nonnormalized cross correlation. The estimated coupling factor $\hat{\lambda}$ is then computed by

$$
\hat{\lambda}=\frac{\chi_{\max }}{\sum_{i} \theta\left(t_{i}\right)^{2}}
$$

where $\theta\left(t_{i}\right)$ denotes the pointing angle measured at time $t_{i}$, and the sum is taken over all values recorded during the maneuver. The estimated time shift between the two time series is a by-product of this method.

The Bayesian approach can be sketched as follows. Let $\lambda=$ $\left\{\lambda_{1}, \ldots, \lambda_{6}\right\}$ denote the TTL coupling factors. By Bayes' theorem

$$
\operatorname{PDF}(\lambda \mid \text { data }) \propto \operatorname{PDF}(\lambda) \cdot \operatorname{PDF}(\text { data } \mid \lambda)
$$

where 1) data denote the information contained in the measurements of range and pointing angles; 2) $\operatorname{PDF}(\lambda \mid$ data) is the probability density function of $\lambda$, given the data; 3) $\operatorname{PDF}(\lambda)$ is the prior of $\lambda$; and 4) $\operatorname{PDF}($ data $\mid \lambda)$ is the likelihood of the data, given $\lambda$.
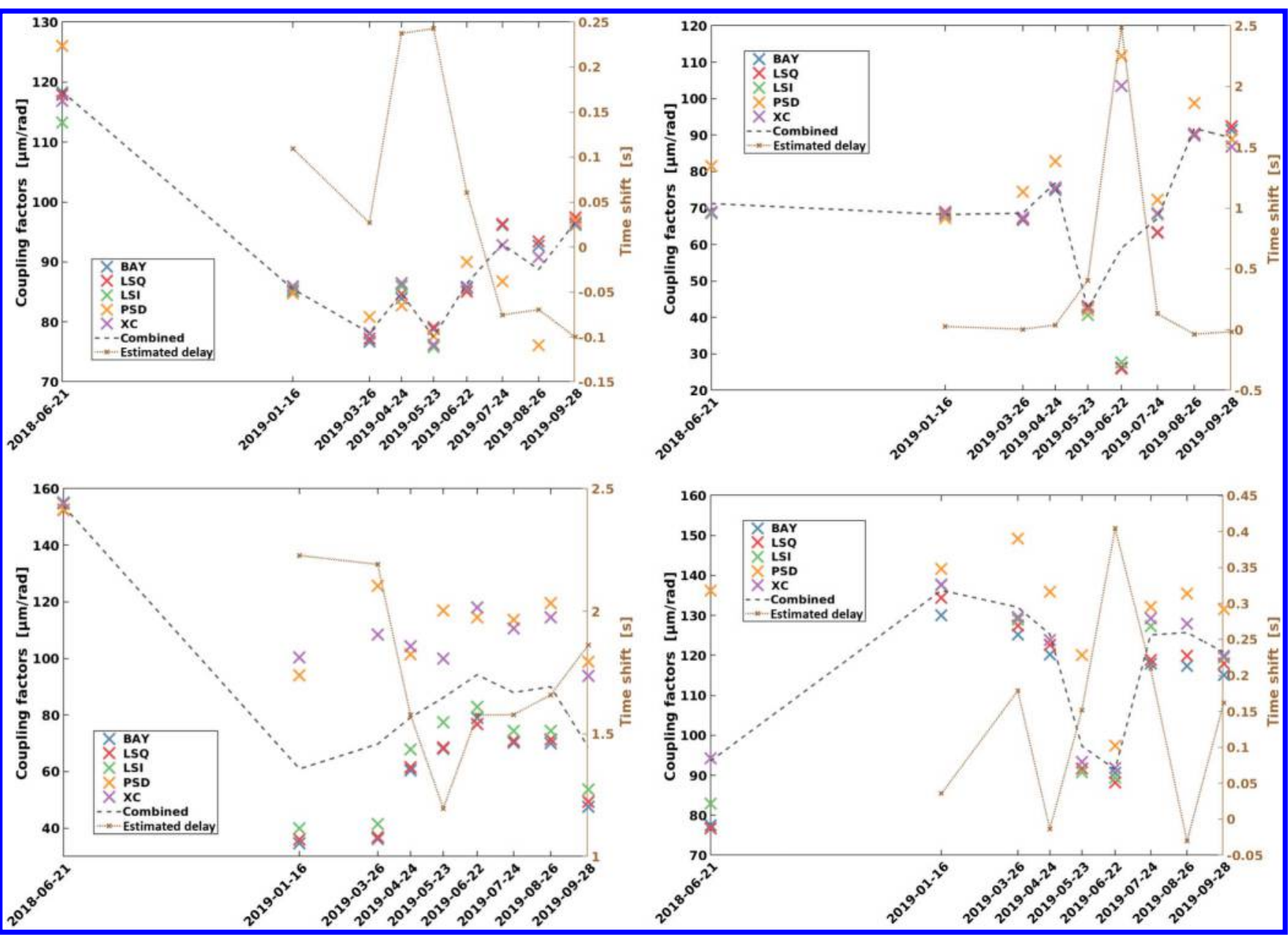

Fig. 8 Estimated TTL coupling factors for GF1 (left) and GF2 (right) for pitch (top) and yaw (bottom), comparing different parameter estimation methods. 
The right-hand side can be computed, where $\operatorname{PDF}(\lambda) \equiv 1$ can be set, if nothing is known about the prior. An estimator of $\lambda$ can be defined by maximizing this expression with respect to $\lambda$. The probability that $\lambda$ lies in a certain region $S \subset \mathbb{R}^{6}$ is given by

$$
P(\lambda \in S)=\int_{S} \operatorname{PDF}(\lambda \mid \text { data }) \mathrm{d} \lambda=\frac{1}{F} \int_{S} \operatorname{PDF}(\lambda) \cdot \operatorname{PDF}(\text { data } \mid \lambda) \mathrm{d} \lambda
$$

where

$$
F=\int_{\mathbb{R}^{6}} \operatorname{PDF}(\lambda) \cdot \operatorname{PDF}(\text { data } \mid \lambda) \mathrm{d} \lambda
$$

is the proportionality factor for Eq. (6). Through the term $\operatorname{PDF}($ data $\mid \lambda$ ), any additional information (e.g., on the noise of the data) can be considered.

Figure 8 shows a comparison of the pitch and yaw coupling factors estimated by different methods. Note that the changes for
GF1 between June 2018 and January 2019 are mainly due to a mass trim maneuver carried out on 18 July 2018. The large differences for the yaw GF1 angle have the following reason. The pointing angle excitations during all yaw GF1 maneuvers appear to be time shifted with respect to the excitations in the biased range measured by the LRI, independent of which attitude sensor is used. A similar effect is observed for the pitch GF2 maneuvers performed on 22 June 2019. The cause of this observation is currently still under investigation. In these cases, the PSD and XC methods tend to yield larger values for the estimated coupling factors, because the PSD method is insensitive to time stamp mismatches, and the XC method accounts for the time shift and estimates it along with the coupling factor. The estimated time shifts are also depicted in Fig. $\underline{8}$, in which each value is the mean of two time shifts estimated from two maneuvers. In Fig. 9, the yaw GF1 time shift is visible in the time domain (bottom left plot). The angles shown in Figs. $\underline{8}$ and $\underline{9}$ were derived from IMU1B data, but SCA1B or FSM data could be used to produce very similar plots.
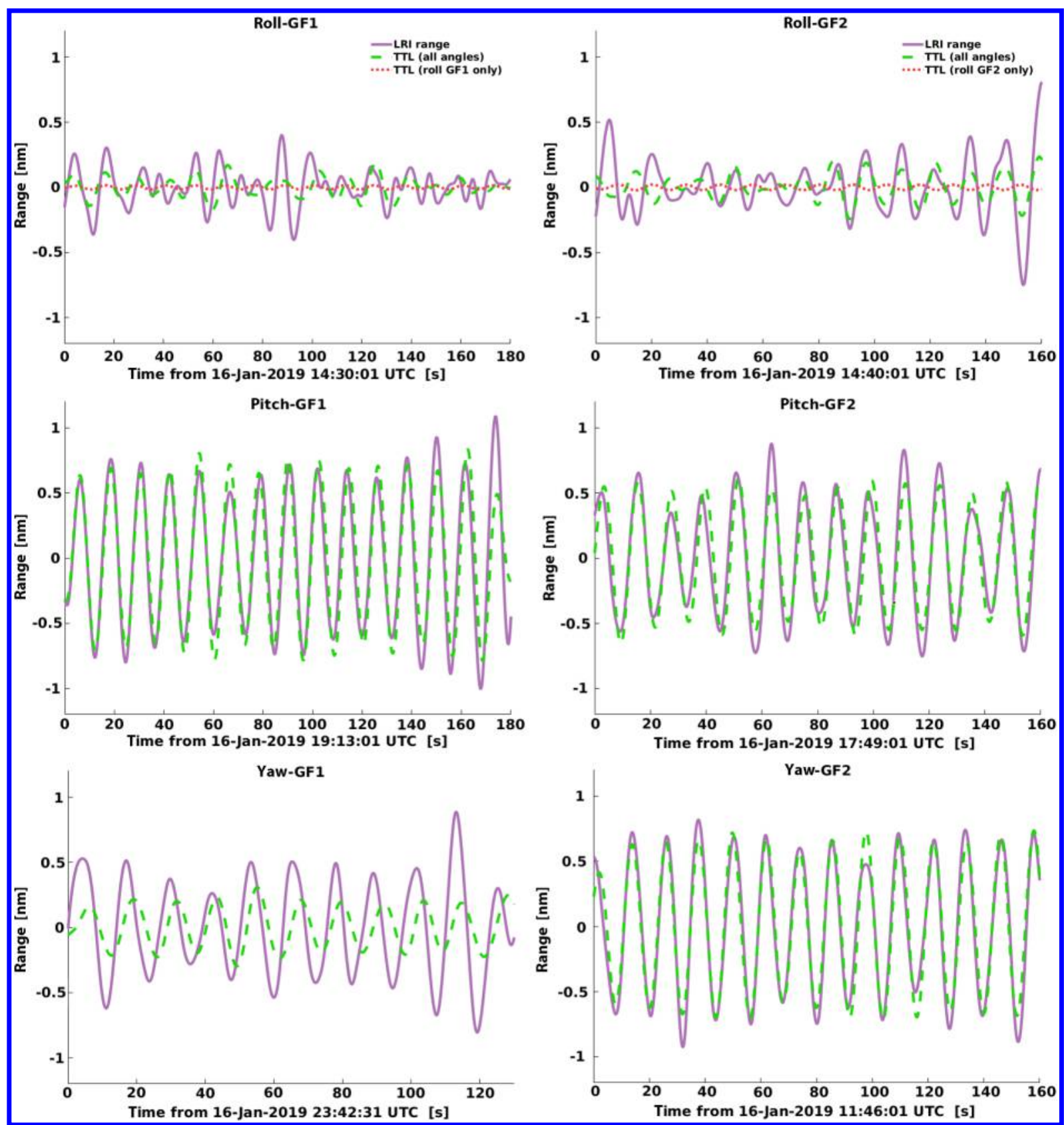

Fig. 9 Band-pass-filtered range vs. fitted TTL for roll (top), pitch (middle) and yaw (bottom) CMC maneuvers in January 2019 for GF1 (left) and GF2 (right). 


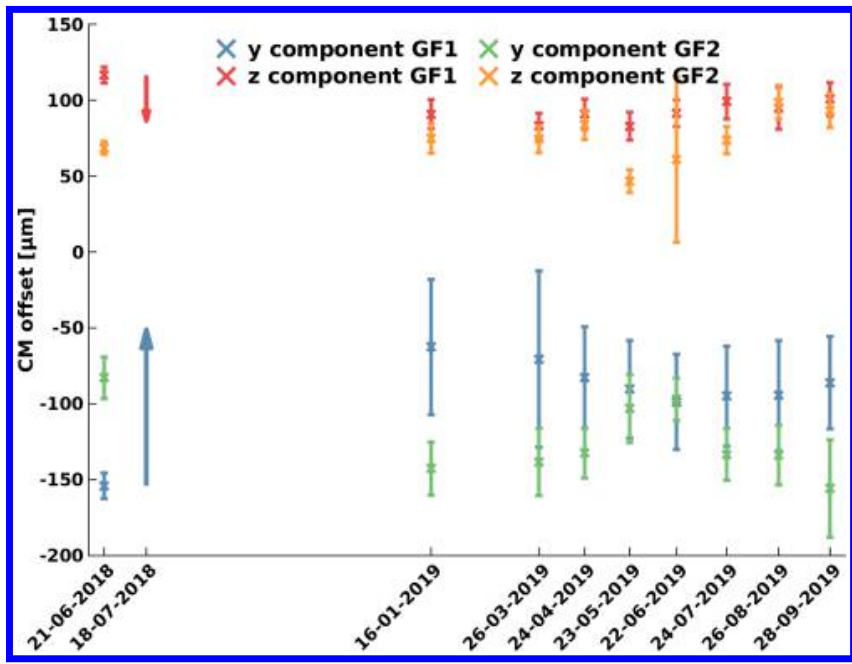

Fig. 10 Estimated $y$ and $z$ components of c.m. positions for GF1 (blue and red) and GF2 (green and orange) with respect to LRI reference point, given in SF.

\section{Results}

By the time of writing, eight CMC maneuvers have been performed while the LRI was in science mode. Wiggle maneuvers, which are similar to CMC maneuvers, but with a duration of 24 instead of $180 \mathrm{~s}$, have been performed in June 2018. Only one of these short maneuvers was performed per S/C angle, such that the uncertainty of these values is larger. Figure 9 examplarily shows the fitted TTL error for CMC maneuvers performed in January 2019. It is also illustrated that the part of the TTL error corresponding to the roll angle is negligible (top plots, dotted red line). All estimation results are summarized in Table 1. Each row in Table 1 denotes not a single, but an entire set of maneuvers, consisting of $\overline{1} 4$ maneuvers for each CMC and 6 maneuvers for the wiggle. A set of $14 \mathrm{CMC}$ maneuvers contains two mixed maneuvers, one per S/C. The values for pitch and yaw coupling factors are averages over the estimates from the five different methods described previously. Mixed maneuvers, as well as wiggle maneuvers, were only considered by the methods LSQ and BAY. Roll coupling factors were only estimated with the methods LSQ and BAY. The values are also averaged over the estimates from different pointing angle sources: SCA1B RL04, FSM, and IMU1B RL04. Angles derived from ACCs have not been used for the results shown here because the quality of the ACC data was not consistent over the entire investigated time span.

According to Eq. (4), Table 1 also shows our estimations of the SF $y$ and $z$ components of the S/C c.m. positions with respect to the VP. The estimated c.m. positions are plotted in Fig. 10. On 18 July 2018, a mass trim maneuver was performed on GF1. That is, the c.m. of GF1 was shifted, using movable trim masses on the $\mathrm{S} / \mathrm{C}$, to a point which had been estimated to be the ACC reference point. In Fig. 10, the SF $y$ and $z$ components of these mass shifts are shown as arrows.

The formal errors of the least-squares fit, derived from Eq. (5), are valid under ideal assumptions, but likely underestimate the uncertainty of the parameters estimated from real data. For this reason, the values in Table 1 are presented in the form $\mu \pm \sigma$, where $\mu$ is the average value over a set of values derived from different methods and different pointing angle sources, as mentioned previously, and $\sigma$ is the standard deviation of this set of values. The same uncertainties have been used in Fig. 10.

\section{Conclusions}

The preflight requirements on the LRI linear TTL coupling factors per S/C were 20,200 , and $200 \mu \mathrm{m} \cdot \mathrm{rad}^{-1}$ for roll, pitch, and yaw, respectively. The authors conclude that these requirements are met. Gravity field recovery from LRI range should not be limited by TTL in any frequency band. Because the LRI provides pitch and yaw angles with good accuracy, the estimation of the coupling factors can be performed without any auxiliary data. However, the authors recap that gyroscope or star camera data may be used in place of steering mirror data, providing very similar results. Different estimation methods have been tested successfully and presented here. The pointing angles largely fit the observed range very well, with the exception of yaw GF1 angles, which is due to an apparent time stamp mismatch and subject to further investigation.

It has been shown that the activation of attitude control thruster firings produces a linear $\mathrm{S} / \mathrm{C}$ acceleration, which is measured by the LRI. These disturbances can be modeled and removed from the range in order not to falsify the TTL estimation. It is noteworthy, however, that an imperfect response model might introduce some error to the parameter estimation. This could potentially be mitigated by downweighting observations close to a thruster firing. In general, weighting based on signal quality indicators, such as the signal-to-noise ratio of the phase readout, could be beneficial, but is beyond the scope of this paper.

Although the CMC maneuvers are not optimized for LRI TTL calibration, it has been demonstrated here that they can be used for this purpose and yield good results. For future satellite geodesy missions, where other noise contributors may be suppressed to a larger extent and the requirements on the ranging noise may be stricter, one may consider to perform dedicated calibration maneuvers.

The data support the assumption that the offset between LRI VP and $\mathrm{S} / \mathrm{C}$ c.m. is the main effect of TTL coupling in the LRI measurements. The coupling factors correspond to nadir and cross-track components of the S/C c.m. Therefore, the analysis also provides estimations of S/C c.m. movement over time, excluding the along-track direction. The results correctly depict the c.m. shifts of the mass trims performed on 18 July 2018 . The observed variations of the estimated coupling factors likely reflect small fluctuations of the S/C c.m. The potential of estimating c.m. positions by means of LRI data is especially useful, because the ACC data, which are nominally used for this purpose, are, on GF2, currently not available with the highest quality.

\section{Appendix: Notation for the Dynamics Equations}

The following notation is used in Eqs. (2) and (ㄱ) :

1) The attitude quaternions are denoted by $\boldsymbol{q}=\left(q_{0}, q_{i}, q_{j}, q_{k}\right)^{T}$, where $q_{0}$ is the real part, describing the rotation angle, and $\left(q_{i}, q_{j}, q_{k}\right)^{T}$ is the imaginary part, describing the rotation axis.

2) The $S / C$ angular velocities in satellite frame are denoted by $\omega$.

3) $\Omega(\omega)$ is a $4 \times 4$ matrix given by

$$
\Omega(\boldsymbol{\omega})=\left(\begin{array}{cccc}
0 & -\omega_{x} & -\omega_{y} & -\omega_{z} \\
\omega_{x} & 0 & \omega_{z} & -\omega_{y} \\
\omega_{y} & -\omega_{z} & 0 & \omega_{x} \\
\omega_{z} & \omega_{y} & -\omega_{x} & 0
\end{array}\right)
$$

4) The total torque acting on the satellite is denoted by $\tau$.

5) $J$ denotes the satellite moment of inertia (MOI).

The MOI for GRACE-FO is given in [24]. The MOI for GRACE can be found in [20].

\section{Acknowledgments}

Parts of this work have been financially supported by the Bundesministerium für Bildung und Forschung, project number 03F0654B. Moreover, parts of this work have been supported by funding from the Sonderforschungsbereich 1128 "Relativistic Geodesy and Gravimetry with Quantum Sensors (geo-Q)" by the Deutsche Forschungsgemeinschaft. Furthermore, the authors would like to thank the Max Planck Society (Max-Planck-Gesellschaft) for support within the LEGACY framework on low-frequency gravitational wave astronomy (M.IF.A.QOP18098). The authors would like to thank the Gravity Recovery and Climate Experiment Follow-On Science Data System (SDS) for providing Level-1 instrument data. Furthermore, the authors would like to thank the German Space Operations Center (DLR, German Aerospace CenterGSOC) for helpful discussions about GRACE Follow-On attitude control. 


\section{References}

[1] Tapley, B. D., Bettadpur, S., Watkins, M. M., and Reigber, C., "The Gravity Recovery and Climate Experiment: Mission Overview and Early Results," Geophysical Research Letters, Vol. 31, No. 9, 2004, p. 9607. https://doi.org/10.1029/2004GL019920

[2] Tapley, B. D., Bettadpur, S., Ries, J. C., Thompson, P. F., and Watkins, M. M., "GRACE Measurements of Mass Variability in the Earth System," Science, Vol. 305, No. 5683, 2004, pp. 503-505.

https://doi.org/10.1126/science.1099192

[3] Wouters, B., Bonin, J. A., Chambers, D. P., Riva, R. E. M., Sasgen, I., and Wahr, J., "GRACE, Time-Varying Gravity, Earth System Dynamics and Climate Change," Reports on Progress in Physics, Vol. 77, No. 11, 2014, Paper 116801 https://doi.org/10.1088/0034-4885/77/11/116801

[4] Tapley, B. D., Watkins, M. M., Flechtner, F., Reigber, C., Bettadpur, S., Rodell, M., Sasgen, I., Famiglietti, J. S., Landerer, F. W., Chambers, D. P., Reager, J. T., Gardner, A. S., Save, H., Ivins, E. R., Swenson, S. C., Boening, C., Dahle, C., Wiese, D. N., Dobslaw, H., Tamisiea, M. E., and Velicogna, I., "Contributions of GRACE to Understanding Climate Change," Nature Climate Change, Vol. 9, No. 5, 2019, pp. 358-369. https://doi.org/10.1038/s41558-019-0456-2

[5] Kornfeld, R. P., Arnold, B. W., Gross, M. A., Dahya, N. T., Klipstein, W. M., Gath, P. F., and Bettadpur, S., "GRACE-FO: The Gravity Recovery and Climate Experiment Follow-On Mission," Journal of Spacecraft and Rockets, Vol. 56, No. 3, 2019, pp. 931-951. https://doi.org/10.2514/1.A34326

-[6] Kim, J., and Lee, S. W., "Flight Performance Analysis of GRACE KBand Ranging Instrument with Simulation Data," Acta Astronautica, Vol. 65, Nos. 11-12, 2009, pp. 1571-1581. https://doi.org/10.1016/j.actaastro.2009.04.010

-[7] Abich, K., Abramovici, A., Amparan, B., Baatzsch, A., Okihiro, B. B., Barr, D. C., Bize, M. P., Bogan, C., Braxmaier, C., Burke, M. J., Clark, K. C., Dahl, C., Dahl, K., Danzmann, K., Davis, M. A., de Vine, G., Dickson, J. A., Dubovitsky, S., Eckardt, A., Ester, T., Barranco, G. F., Flatscher, R., Flechtner, F., Folkner, W. M., Francis, S., Gilbert, M. S., Gilles, F., Gohlke, M., Grossard, N., Guenther, B., Hager, P., Hauden, J., Heine, F., Heinzel, G., Herding, M., Hinz, M., Howell, J., Katsumura, M., Kaufer, M., Klipstein, W., Koch, A., Kruger, M., Larsen, K., Lebeda, A., Lebeda, A., Leikert, T., Liebe, C. C., Liu, J., Lobmeyer, L., Mahrdt, C., Mangoldt, T., McKenzie, K., Misfeldt, M., Morton, P. R., Müller, V., Murray, A. T., Nguyen, D. J., Nicklaus, K., Pierce, R., Ravich, J. A., Reavis, G., Reiche, J., Sanjuan, J., Schütze, D., Seiter, C., Shaddock, D., Sheard, B., Sileo, M., Spero, R., Spiers, G., Stede, G., Stephens, M., Sutton, A., Trinh, J., Voss, K., Wang, D., and Wang, R. T., "In-Orbit Performance of the GRACE Follow-On Laser Ranging Interferometer," Physical Review Letters, Vol. 123, No. 3, 2019, Paper 031101. https://doi.org/10.1103/PhysRevLett.123.031101

-[8] Sheard, B. S., Heinzel, G., Danzmann, K., Shaddock, D. A., Klipstein, W. M., and Folkner, W. M., "Intersatellite Laser Ranging Instrument for the GRACE Follow-On Mission," Journal of Geodesy, Vol. 86, No. 12, 2012, pp. 1083-1095. https://doi.org/10.1007/s00190-012-0566-3

[9] Heinzel, G., Sheard, B., Brause, N., Danzmann, K., Dehne, M., Gerberding, O., Mahrdt, C., Müller, V., Schütze, D., Stede, G., Klipstein, W., Folkner, W., Spero, R., Nicklaus, K., Gath, P., and Shaddock, D., "Laser Ranging Interferometer for GRACE Follow-On," SPIE Proceedings of the International Conference on Space Optics-ICSO 2012, Vol. 10564, Soc. of Photo-Optical Instrumentation Engineers, Bellingham, Washington, 2017, pp. 517-526. https://doi.org/10.1117/12.2309099

[10] Abich, K., Bogan, C., Braxmaier, C., Danzmann, K., Dehne, M., Gohlke, M., Görth, A., Heinzel, G., Herding, M., Mahrdt, C., Müller, V., Nicklaus, K., Sanjuan, J., Schütze, D., Sheard, B., Stede, G., and Voss, K., "GRACE-Follow On Laser Ranging Interferometer: German Contribution," Journal of Physics: Conference Series, Vol. 610, No. 12, 2015, Paper 012010.

https://doi.org/10.1088/1742-6596/610/1/012010

[11] Schütze, D., Müller, V., Stede, G., Sheard, B. S., Heinzel, G., Danzmann, K., Sutton, A. J., and Shaddock, D. A., "Retroreflector for GRACE Follow-On: Vertex vs. Point of Minimal Coupling," Optics Express, Vol. 22, No. 8, 2014, pp. 9324-9333. https://doi.org/10.1364/OE.22.009324

[12] Schütze, D., "Intersatellite Laser Interferometry: Test Environments for GRACE Follow-On,” Ph.D. Thesis, Leibniz Universität Hannover, Hannover, Germany, 2015. https://doi.org/10.15488/8576
[13] Fleddermann, R., Ward, R. L., Elliot, M., Wuchenich, D. M., Gilles, F., Herding, M., Nicklaus, K., Brown, J., Burke, J., Dligatch, S., Farrant, D. I., Green, K. L., Seckold, J. A., Blundell, M., Brister, R., Smith, C., Sheard, B. S., Heinzel, G., Danzmann, K., Klipstein, B., McClelland, D. E., and Shaddock, D. A., "Testing the GRACE Follow-On Triple Mirror Assembly," Classical and Quantum Gravity, Vol. 31, No. 19, 2014, Paper 195004.

https://doi.org/10.1088/0264-9381/31/19/195004

[14] Müller, V., "Design Considerations for Future Geodesy Missions and for Space Laser Interferometry," Ph.D. Thesis, Leibniz Universität Hannover, Hannover, Germany, 2017.

https://doi.org/10.15488/9029

[15] Wanner, G., and Karnesis, N. A., "Preliminary Results on the Suppression of Sensing Cross-Talk in LISA Pathfinder," Journal of Physics: Conference Series, Vol. 840, 2017, Paper 012043. https://doi.org/10.1088/1742-6596/840/1/012043

[16] Chwalla, M., Danzmann, K., Alvarez, M. D., Esteban Delgado, J. J., Fernández Barranco, G., Fitzsimons, E., Gerberding, O., Heinzel, G., Killow, C. J., Lieser, M., Perreur-Lloyd, M., Robertson, D., I, Schuster, S., Schwarze, T. S., Tröbs, M., Wanner, G., Ward, H., and Zwetz, M., "Optical Suppression of Tilt-to-Length Coupling in the LISA LongArm Interferometer," arXiv e-prints, 2020. https://doi.org/10.1103/PhysRevApplied.14.014030

-[17] Flechtner, F., Neumayer, K.-H., Dahle, C., Dobslaw, H., Fagiolini, E., Raimondo, J.-C., and Güntner, A., "What Can Be Expected from the GRACE-FO Laser Ranging Interferometer for Earth Science Applications?" Surveys in Geophysics, Vol. 37, March 2016, pp. 453-470.

[18] Touboul, P., Foulon, B., and Willemenot, E., "Electrostatic Space Accelerometers for Present and Future Missions," Acta Astronautica, Vol. 45, No. 10, 1999, pp. 605-617. https://doi.org/10.1016/S0094-5765(99)00132-0

- [19] Frommknecht, B., Oberndorfer, H., Flechtner, F., and Schmidt, R., "Integrated Sensor Analysis for GRACE-Development and Validation," Advances in Geosciences, Vol. 1, 2003, pp. 57-63. https://doi.org/10.5194/adgeo-1-57-2003

[20] Wang, F., "Study on Center of Mass Calibration and K-Band Ranging System Calibration of the GRACE Mission," Ph.D. Thesis, Univ. of Texas at Austin, Austin, TX, 2003.

[21] Wang, F., Bettadpur, S., Save, H., and Kruizinga, G., "Determination of Center-of-Mass of Gravity Recovery and Climate Experiment Satellite," Journal of Spacecraft and Rockets, Vol. 47, No. 2, 2010, pp. 371-379. https://doi.org/10.2514/1.46086

[22] Herman, J., Presti, D., Codazzi, A., and Belle, C., "Attitude Control for GRACE," Proceedings of the 18th International Symposium on Space Flight Dynamics, Deutsches Zentrum für Luft- und Raumfahrt, Cologne, Germany, 2004, p. 27.

[23] Gath, P., "Entwicklung, Integration und Test der GRACE Follow-On Satelliten," Deutsche Gesellschaft für Luft- und Raumfahrt - LilienthalOberth e.V., Bonn, 2016.

[24] Wen, H. Y., Kruizinga, G., Paik, M., Landerer, F., Bertiger, W., Sakumura, C., Bandikova, T., and McCullough, C., "Gravity Recovery and Climate Experiment Follow-On (GRACE-FO) Level-1 Data Product User Handbook," NASA Jet Propulsion Lab., California Inst. of Technology, TR JPL D-56935, Sept. 2019, https://podaac.jpl.nasa.gov/GRACE-FO.

-[25] Kayali, S., Morton, P., and Gross, M., "International Challenges of GRACE Follow-On,” 2017 IEEE Aerospace Conference, IEEE Publ., Piscataway, NJ, 2017, pp. 1-8. https://doi.org/10.1109/AERO.2017.7943615

[26] McCullough, C. M., Harvey, N., Save, H., and Bandikova, T., "Description of Calibrated GRACE-FO Accelerometer Data Products (ACT) (v. 04)," Jet Propulsion Lab., California Inst. of Technology, and Center for Space Research, Univ. of Texas at Austin, TR JPL D-103863, Austin, TX, May 2019, https://podaac.jpl.nasa.gov/GRACE-FO.

[27] Bandikova, T., Flury, J., and Ko, U.-D., "Characteristics and Accuracies of the GRACE Inter-Satellite Pointing," Advances in Space Research, Vol. 50, No. 1, 2012, pp. 123-135.

[28] Wertz, J. R., Spacecraft Attitude Determination and Control, Kluwer Academic, Norwell, MA, 1978, p. $150 \mathrm{ff}$.

[29] Tröbs, M., and Heinzel, G., "Improved Spectrum Estimation from Digitized Time Series on a Logarithmic Frequency Axis," Measurement, Vol. 39, No. 2, 2005, pp. 120-129. https://doi.org/10.1016/j.measurement.2005.10.010

[30] Müller, V., "Simulations for LISA and GRACE Follow-On," Master's Thesis, Leibniz Universität Hannover, Hannover, Germany, 2013.

[31] Darbeheshti, N., Wegener, H., Müller, V., Naeimi, M., Heinzel, G., and Hewitson, M., "Instrument Data Simulations for GRACE Follow-On: Observation and Noise Models," Earth System Science Data, Vol. 9, No. 2, 2017, pp. 833-848. https://doi.org/10.5194/essd-9-833-2017 
[32] Misfeldt, M., "Data Processing and Investigations for the GRACE Follow-On Laser Ranging Interferometer,' Master's Thesis, Leibniz Universität Hannover, Hannover, Germany, 2019. https://doi.org/10.15488/9639

[33] Heinzel, G., Rüdiger, A., and Schilling, R., "Spectrum and Spectral Density Estimation by the Discrete Fourier Transform (DFT), Including a Comprehensive List of Window Functions and Some New Flat-Top Windows," Max Planck Inst., 2002, http://hdl.handle.net/11858/00001M-0000-0013-557B-3.

O. Abdelkhalik Associate Editor 